\title{
Histoplasmosis in the Lung of a Race Horse with Yersiniosis
}

\author{
Yoshinari KATAYAMA $^{1)}$, Atsutoshi KUWANO ${ }^{2)}$ and Toyohiko YOSHIHARA ${ }^{2)}$ \\ ${ }^{1)}$ Microbiology Division, Epizootic Research Station, Equine Research Institute, Japan Racing Association, 1400-4 Shiba, Kokubunji- \\ machi, Shimotsuga-gun, Tochigi 329-0412 and ${ }^{2}$ Clinical Sciences \& Pathobiology Division, Equine Research Institute, Japan Racing \\ Association, 321-4 Tokami-cho, Utsunomiya, Tochigi 320-0856, Japan
}

(Received 28 September 2000/Accepted 16 July 2001)

ABSTRACT. A 4-year-old female thoroughbred race horse died of acute peritonitis caused by necrotizing granulomatous duodenitis. Yersinia enterocolitica was immunohistochemically demonstrated in macrophages in granulomas developed in the duodenum, lung, liver and abdominal lymph nodes. The yeast-like fungi were found in the cytoplasmic vacuoles of macrophages in the lung that infiltrated into the granulomas and surrounding alveoli with congestive edema. The yeast-like fungi were positively stained by Gomori-Grocott chromic acid methenamine silver stain and immuno-histochemically stained with anti-histoplasma antibody. In this case, it was considered that granulomas formed in the duodenum, lung, liver and abdominal lymph nodes were primarily caused by Yersinia enterocolitica due to idiopathic weakening of the immune system. Yeast-like fungi immunohistochemically identified as histoplasmas secondarily infected the lung. This is the first case regarded as equine histoplasmosis capsulati in Japan.

KEY WORDS: equine, histoplasmosis, Yersinia enterocolitica.

Histoplasmosis is a disease characterized by granuloma in the lung and has been reported in humans and other animals. Histoplasma capsulatum (H. capsulatum), a causal agent of histoplasmosis, is a dimorphic fungus found in the soil and transmitted by bats. The disease is endemic to the United States, and is especially common in the Mississippi, Ohio and St. Lawrence River Valley areas [5]. A few cases have been reported among tourists in Japan [3], however, there is no report of $H$. capsulatum isolated from soil in Japan. We encountered a race horse with yersiniosis in the duodenum, lung, liver and mesenteric lymph node, and histoplasmosis in the lung, and the present paper describes the histopathological and immunohistochemical characteristics of the lesions.

The 4-year-old, female thoroughbred was born to a French horse that had been living in Japan for four years in Hokkaido. After participating in twenty races, the horse demonstrated symptoms of fatigue, anemia, fever, abnormal breath sound, and anorexia. The horse was treated mainly with antibiotics, but did not improve. The horse continuously lost weight and died after 2 months. There were no abnormal findings except for a decrease in white and red blood cell counts observed on a hematological examination during an affected period. At necropsy, there was a finding of acute peritonitis due to duodenitis with perforation. Other gross findings were congestion or hemmorhage of all lobes and albedo nodes of $0.5-1.0 \mathrm{~cm}$ in diameter sporadically developed in the lung. Mesentric, hepatic, splenic and pulmonary lymph nodes showed moderate swelling. On bacteriological examination of the duodenum and liver, Escherichia coli and Streptococcus sp. were isolated from both organs. For histopathological examination the sliced tissue blocks were fixed with $20 \%$ neutral buffered formalin, dehydrated with ethanol, and embedded in paraffin. Sections of approximately $4 \mu \mathrm{m}$ thick were processed for hematoxylin and eosin (H\&E), periodic acid-Schiff's (PAS) reaction, Gomori-Grocott chromic acid methenamine silver (GMS), Ziehl-Neelsen, Gram and mucicarmine stains. For immunohistochemical examination (avidin-biotin-peroxidase complex procedure; Vectastain Elite ABC kit, Vector Labs, CA), paraffin sections of the lung, duodenum, liver and mesenteric lymph node were reacted with rabbit antihistoplasma antibody (Meridian Daiagnostics, Inc., U.S.A.)[2], mouse anti-Pneumocystis carinii (P. carinii) antibody (Dako, Denmark), mouse anti- $Y$. enterocolitica O:3 (Progen Biotechnik, GMBH) or O:9 (Progen Biotechnik, GMBH) antibody, respectively.

Histopathologically, the albedo nodes of the lung observed macroscopically showed granuloma with degenerated tissue in the center. Macrophages, lymphocytes, multinucleated giant cells, plasma cells, epithelioid cells and fibroblasts were seen in the peripheral area (Fig. 1). Extensive congestive edema was observed around the granuloma in the lung. A large number of macrophages with numerous vacuoles in the cytoplasm were observed in the granuloma as well as its surrounding alveoli and interstitial tissue. In vacuoles of macrophages, H\&E-stained acidophilic or weak basophilic globular bodies with a diameter of 1-3 $\mu \mathrm{m}$ were observed (Fig. 2). The small globular bodies were stained positively with GMS (Fig. 3) and showed a slightly positive with PAS reaction. The globular bodies were negative with Gram staining and mucicarmine staining. Acid fast bacteria were not detected in macrophages containing globular bodies by the method of Ziehl-neelsen. Therefore, the small globular bodies were considered yeast-like fungi.

In the perforated duodenum, liver, and mesentric lymph nodes, there were granulomas elaborated with degenerating macrophages in the center and macrophages, lymphocytes, plasma cells and fibroblasts in the peripheral area (Fig. 4). No small globular bodies were observed in the cytoplasm of 

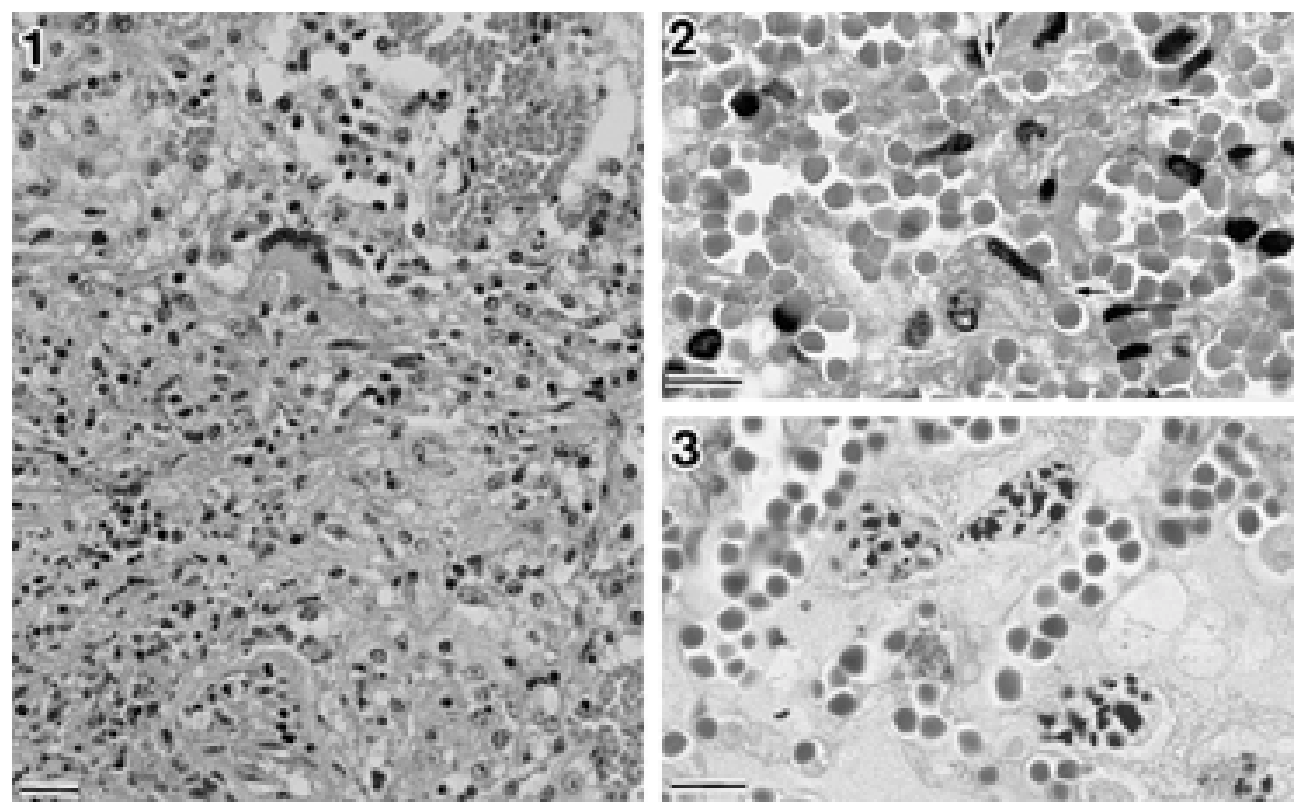

Fig. 1. Granuloma in the lung. Macrophages, lymphocytes, multinucleated giant cells and epithelioid cells observed around the periphery of the necrotic granuloma (H\&E stain; bar, $20 \mu \mathrm{m}$ ).

Fig. 2. Congestion and edema in the lung. Arrows show small globular bodies in the cytoplasm of the macrophages (H\&E stain; bar, $10 \mu \mathrm{m}$ ).

Fig. 3. Yeast-like fungi in the cytoplasma of macrophages stained positively with Gomori-Grocott chromic acid methenamine silver-nitrate stain (bar; $10 \mu \mathrm{m}$ ).

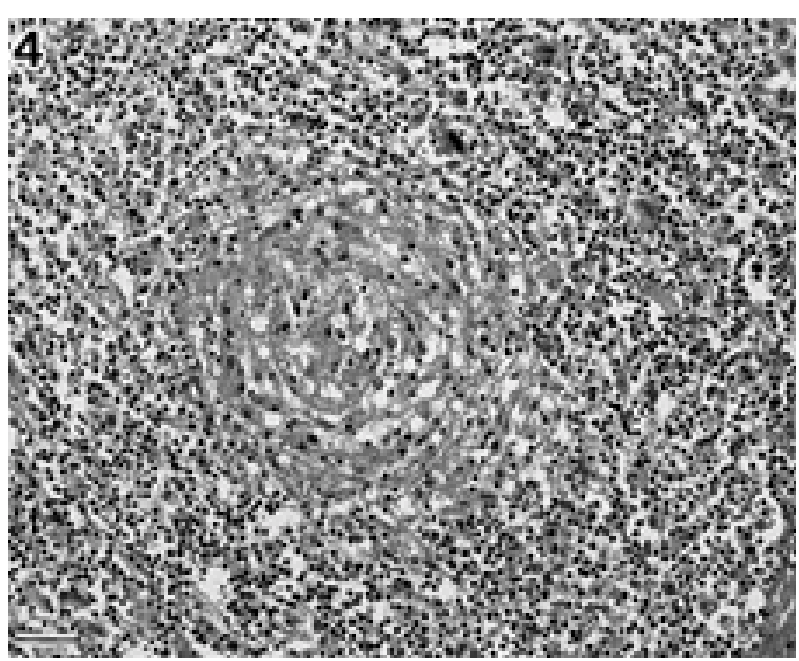

Fig. 4. Granuloma in the duodenum. Macrophages, lymphocytes, multinucleated giant cells, plasma cells and epithelioid cells observed around the periphery of the necrotic center (H\&E stain; bar, $40 \mu \mathrm{m})$.

macrophages in these granulomas. The lymphatic follicles in the lymph nodes of the body surface and those in the spleen were atrophic.

Immunohistochemically, small globular bodies in the macrophages infiltlating in the granuloma and in the lung with congestive edema reacted positively to anti-histo- plasma antibody (Fig. 5) but did not react to anti-P. carinii and anti-Y. enterocolitica $\mathrm{O}: 9$ antibodies. The cytoplasm of macrophages in the granuloma in the lung, duodenum, liver and mesenteric lymph node reacted positively to anti- $Y$. enterocolitica $\mathrm{O}: 3$ antibody (Fig. 6).

Therefore, it was thought that the cause of death was complications of acute peritonitis resulting from necrosis of the intestinal wall with perforation due to the granuloma formation in the duodenum by $Y$. enterocolitica. In addition, histoplasma and $Y$. enterocolitica caused mixed infection of the lung. As granulomas were formed by $Y$. enterocolitica in several organs, we considered that $Y$. enterocolitica infection was primary, and histoplasma infection was secondary. $Y$. enterocolitica is often found as the causal bacterium in cases of food poisoning in humans, and it usually exists in the digestive tract of normal domestic animals [1]. There is a report that $Y$. enterocolitica is one of the pathogens causing pseudotuberculosis-like lesions in the hare [6]. However, granulomas caused by $Y$. enterocolitica have not previously been reported in the horse. Meantime, it has been reported that the infection of the horse by $H$. capsulatum is associated with a weak immune defense system in juvenile foals or older horses [4]. The present observation was made in an adult horse and the lymphatic organs except for the mesentric, hepatic, splenic and pulmonary lymph nodes were in an atrophic state. The formation of the granuloma in the duodenum, lung, liver and mesenteric lymph node proceeded because of a compromised immune system 

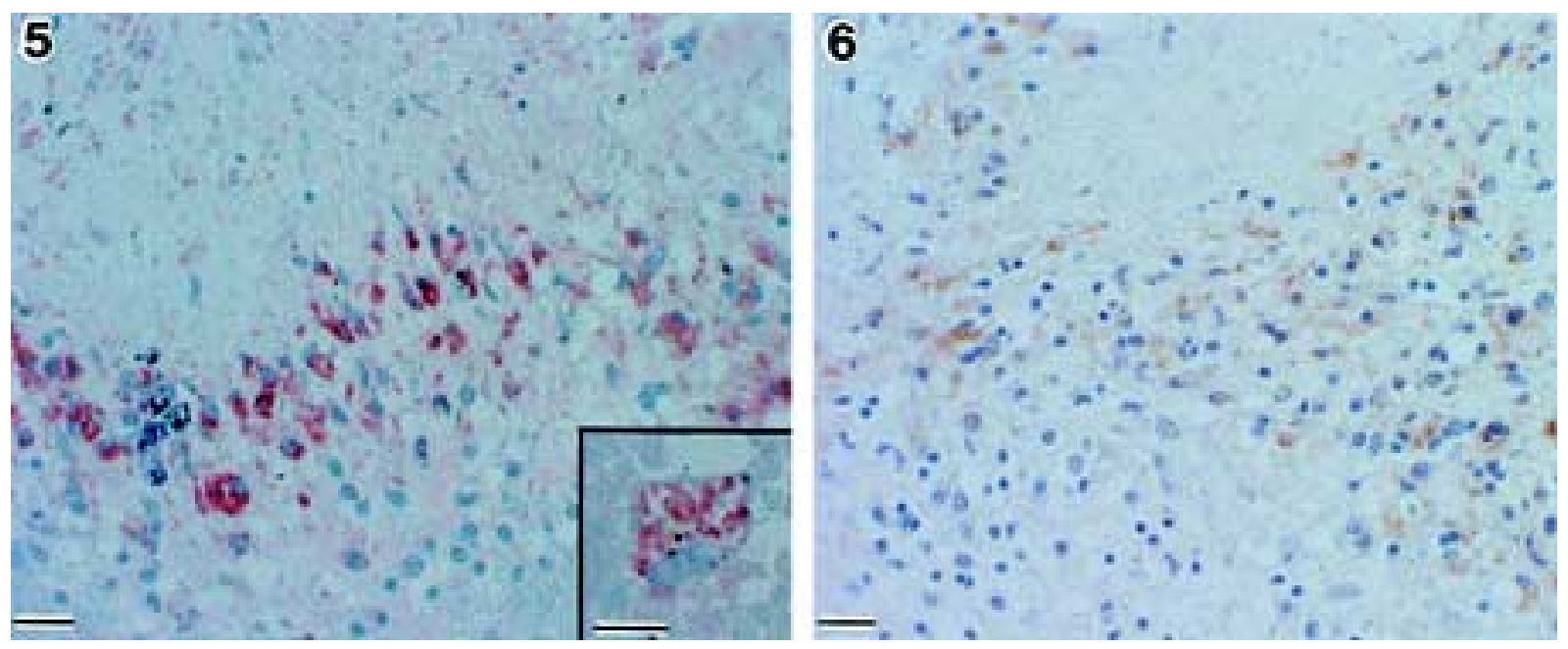

Fig. 5. Immunostaining of the lung. The macrophages positively stained with anti-histoplasma antibody in the granuloma (Hematoxylin counterstain; bar, $20 \mu \mathrm{m}$ ). Inset: The small globular bodies observed in the cytoplasm of a macrophage positively stained by anti-histoplasma antibody in association with congestion and edema (Hematoxylin counterstain; bar, $10 \mu \mathrm{m}$ ).

Fig. 6. Immunostaining of the lung. The macrophages in the lung granuloma reacted positively to anti-Y. enterocolitica O:3 antibody (Hematoxylin counterstain; bar, $20 \mu \mathrm{m}$ ).

with an unknown cause. In this case, histoplasmal infection was confirmed by GMS stain and immunostaining by using anti-histoplasma antibody. There is no reported isolation of $H$. capsulatum from Japanese horse. However, a case report describing infection of dog by H. capsulatum in Japan was presented by Kagawa et al. [2]. Although the mechanism of infection was not clear, this is the first case regarded as equine histoplasmosis in Japan. Recently, many race horses have frequently been transported from foreign countries for participation in races or as stallions or brood mares for breeding. Therefore, there is a possibility that histoplasmosis will be introduced from those horses in Japan. It is necessary to monitor this situation.

ACKNOWLEDGEMENT. The authors would like to thank Dr. Hiroyuki Taniyama, Rakuno Gakuen University, for offer of anti-histoplasma antibody, Dr. Mamoru Yamaguchi and Dr. Mutsumi Miyamoto, Ohio State University, for reviewing the manuscript.

\section{REFERENCES}

1. Browning, G. F., Chalmers, R. M., Snodgrass, D. R., Bat, R. M., Hart, C. A., Ormarod, S. E. Leadon, D., Stoneham, S. J. and Rossdale, P. D. 1991. Equine Vet. J. 23: 405-409.

2. Kagawa, Y., Aoki, S., Iwatomi, T., Yamaguchi, M., Momiyama, N., Hirayama, K. and Taniyama, H. 1998. J. Vet. Med. Sci. 60: 863-865.

3. Miyaji, M. 1991. Pathol. Clin. Med. 14: 1301-1307 (in Japanese).

4. Rezabek, G. B., Donahue, J. M., Giles, R. C. Petrites-Murphy, M. B. Poonacha, J. R., Smith, B. J., Swerczek, T. W. and Tramontin, R. R. 1993. J. Comp. Pathol. 109: 47-55.

5. Vally, V. E. O. 1992. pp. 247-249. In: Pathology of Domestic Animals, 4th ed. vol.3 (Jubb, K. V. F., Kennedy, P. C. and Palmer, N. ed.s), Academic Press, California.

6. Wuthe, H. H. and Aleksic, S. 1997. Berl. Munch. Tierarztl. Wochenshchr. 110: 176-177. 